\title{
Temporally extended retrograde amnesia for spatial information resulting from afterdischarges induced by electrical stimulation of the dorsal hippocampus in mice
}

\author{
CATHERINE LAURENT-DEMIR and ROBERT JAFFARD \\ Université de Bordeaux I, Talence, France
}

\begin{abstract}
The present study was conducted in order to examine whether reversible disruption of hippocampal activity using repeated (four times), spaced (at hourly intervals) electrical stimulation (at current levels producing afterdischarges [ADs]) of the dorsal hippocampus would produce a temporally graded retrograde amnesia for two-choice spatial discrimination problems acquired at different time intervals before treatment. Mice were trained successively on five different two-choice discrimination problems in one of two distinct elevated 8-arm radial mazes (A and B). The first two problems were acquired in Maze A, and the final three problems in Maze B. Hippocampal ADs were elicited approximately $24 \mathrm{~h}$ after the mice had acquired the last discrimination problem (B3). One week later, subjects were tested for retention of all five discrimination problems. Results indicated that, as compared with control groups, experimental subjects were severely impaired for retention of the three discrimination problems acquired from 10 days to 5.5 weeks earlier in Maze B, but showed no deficit for the problems acquired 7 weeks earlier in Maze A. However, for problems acquired in Maze B, no temporal gradient for amnesia was observed, although control mice exhibited significant forgetting with increasing retention intervals. Temporally extended but ungraded retrograde disrupting effect of hippocampal stimulation for discriminations acquired in Maze B is discussed.
\end{abstract}

It is now well established that the hippocampal formation (HF) contributes to memory functions. Surgical removal of the HF produces a selective and severe anterograde amnesia (loss of ability to acquire new memories), combined with some degree of retrograde amnesia (i.e., memory loss for information acquired before the onset of amnesia). In most cases, such retrograde amnesia (RA) has been found to be time dependent: Memory for events that occurred a short time before HF removal is impaired, but very remote memory is spared. Since very remote memories are typically preserved in bitemporal amnesic patients, it is widely considered that the site of permanent memory storage cannot be the HF (for review, see Squire \& Alvarez, 1995).

The existence of a retrograde amnesia but with an identifiable time gradient led to the assumption that memory consolidation (or strengthening of memory across time) can be viewed as a dynamic process that implies a timedependent reorganization in the neuroanatomical substrates that subserve long-term memory for learned information (Squire, 1992, 1994). Several authors have indeed proposed that an interaction must occur between

This research was supported by the CNRS, The University of Bordeaux 1. We thank Thomas Durkin for his help and comments on this manuscript. Correspondence should be addressed to $C$. Laurent-Demir, Laboratoire de Neurosciences Comportementales et Cognitives, Avenue des Facultés, 33405 Talence Cedex, France (e-mail: jaffard@neurocog. u-bordeaux.fr). the HF and sites of long-term memory storage in neocortex in order for memory representations to be formed in an enduring and accessible manner (Miller, 1989; Teyler \& Discenna, 1986). A long temporal gradient for RA ranging from 2 days to 12 weeks has been reported in animals following lesions of the HF (Cho, Béracochéa, \& Jaffard, 1993; Cho \& Kesner, 1996; Kim, Clark, \& Thompson, 1995; Kim \& Fanselow, 1992; Winocur, 1990; ZolaMorgan \& Squire, 1990). Using mice as subjects, previous experiments conducted in our laboratory (Cho et al., 1993) have shown that bilateral ibotenate lesions of the entorhinal cortex produced a lengthy (about 4.5 weeks) and temporally graded retrograde amnesia for spatial discrimination problems, thereby supporting the view that, beyond this 4.5-week period, a hippocampal-independent memory storage system would be capable of mediating the retrieval of information, and, as a corollary, that during the consolidation process the relative contribution of the HF to retrieval processes would gradually diminish. Nevertheless, similar lesion studies in other animals have yielded some inconsistent results. Specifically, results from other studies have failed to reveal a time-dependent gradient (Bolhuis, Stewart, \& Forrest, 1994; Gaffan, 1993; Salmon, Zola-Morgan, \& Squire, 1987), although, as noted, an extended temporal gradient for RA following lesions of the hippocampus (and related structures) has been reported (Cho et al., 1993; Cho \& Kesner, 1996; Kim et al., 1995; Kim \& Fanselow, 1992; Winocur, 1990; Zola-Morgan \& Squire, 1990). Consequently, the ques- 
tion as to whether the HF actually stores memories and/ or mediates the storage of long-term information elsewhere in the brain (neocortex) remains unclear.

Moreover, although studies that have successfully revealed a temporal gradient of retrograde amnesia also have shown that, at some time after acquisition, the hippocampus can be removed without any observable consequence on retention performance, these experiments do not tell us what would have been the contribution of the hippocampus if it still had been present (Nadel, 1991). In order to address the questions of the status of memory storage in the hippocampus (and the time course of the role of the HF in retrieval processes), the use of electrical brain stimulation that reversibly disrupts neuronal activity in specific brain regions (a feature not shared by brain-lesioning techniques) appears to be more appropriate than lesions. Indeed, previous experiments using repeated electroconvulsive shock have reported the existence of a temporally extended time gradient of RA for one-trial passive avoidance learning in mice (Squire \& Spanis, 1984). However, the various experimental para- digms aimed at investigating RA have frequently used a rapidly acquired single-event conditioning procedure. Such procedures do not accurately model the clinical situation in which persons have encountered a multiplicity of relatively separate episodes prior to the traumatic event. Accordingly, in the present experiments, we used the same retrospective method as that used in mice by Cho et al. (1993) and in monkeys by Zola-Morgan and Squire (1990), in which different information of the same type is learned by the same subjects at different time periods before the amnesia-inducing treatment.

Thus, in the present experiment, we assessed the degree of RA for five spatial discrimination problems learned at different time periods prior to repeated induction (four times, $1 \mathrm{~h}$ apart) of ADs produced by electrical stimulation of the dorsal hippocampus in mice. If we accept the hypothesis that the HF sustains memory of such spatial information for a limited time period following acquisition, then hippocampal stimulation should produce amnesia for more recently acquired information with normal retention of more remote information.

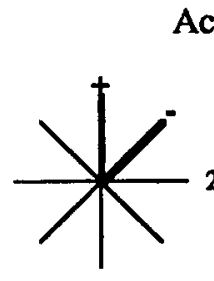

Pair A 1

Acquisition

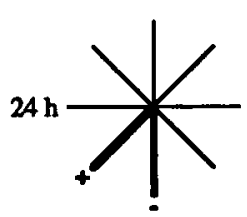

Pair A 2

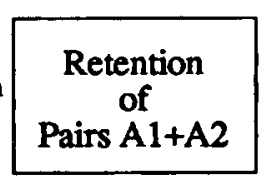

(7 weeks)

\section{Maze A}

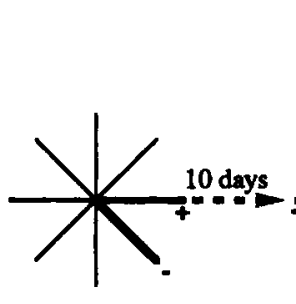

Pair B1

(4.5 weeks)

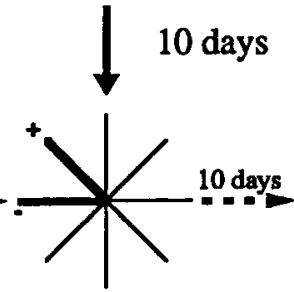

Pair B2

(2.5 weeks)

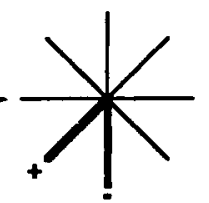

Pair B3

(0.5 weeks)

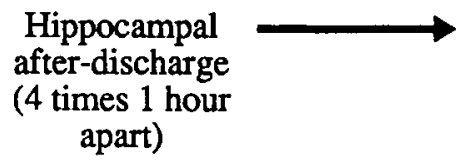

Maze B

\begin{tabular}{|c|}
\hline Retention \\
of 3 pairs \\
in a mixed order \\
\hline
\end{tabular}

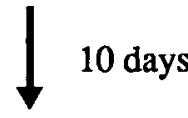

Maze A

\begin{tabular}{|c|}
\hline $\begin{array}{c}\text { Retention } \\
\text { of } \\
2 \text { pairs }\end{array}$ \\
\hline
\end{tabular}

Maze B

\begin{tabular}{|c|}
\hline $\begin{array}{c}\text { Reacquisition } \\
\text { of } 3 \text { pairs } \\
\text { in a mixed order }\end{array}$ \\
\hline
\end{tabular}

Figure 1. Experimental design. Two-choice spatial discriminations involving five different pairs of arms were learned at different time intervals (indicated in parentheses) before elicitation of hippocampal afterdischarges. The first two pairs were learned successively at 24-h intervals in Maze A. Ten days later, mice were subsequently trained on three new successive problems in another maze (B). The three problems were given every 10 days. Amnestic treatment was administrated $24 \mathrm{~h}$ after the mice had acquired the fifth problem in Maze B. 


\section{METHOD}

\section{Animals}

Subjects were 30 male mice of the $\mathrm{Balb} / \mathrm{c} \mathrm{JiCo}$ strain. At 12-16 weeks of age, they were individually housed in cages with ad libitum access to food and water in a constant-temperature room $\left(22^{\circ} \mathrm{C}\right)$ maintained on a 12:12-h light:dark cycle. During training $(0900-1700 \mathrm{~h})$, the mice were placed on a food-deprivation schedule in such a way that their body weights were maintained at $80 \%-85 \%$ of their free-feeding weights.

\section{Apparatus}

Two otherwise identical elevated radial mazes ( $A$ and $B$ ) located in two different rooms decorated with different sets of pictures and objects were used. Each maze was composed of a circular platform (30 cm diameter) from which eight arms $(50 \mathrm{~cm}$ long) radiated in a symmetrical fashion. Automatically operated doors gave access to each arm, the extremity of which was equipped with a food cup.

\section{Behavioral Testing}

Pretreatment training. The overall behavioral protocol is summarized in Figure 1. Basically, the procedure was the same as that previously used by Cho et al. (1993). All mice were successively trained on five different two-choice discrimination problems. Three days before the beginning of learning sessions, the mice were progressively and partially food deprived. At the beginning of the experiment, mice were initially adapted (for a period of about $30 \mathrm{~min}$ ) to the maze by allowing them to freely explore and eat from the food cups at the end of each arm. The following day, training began. Animals were given successive daily sessions composed of 16 trials as follows: For each of the five discrimination problems, two adjacent arms were presented, one of which was always baited (Bioserv. 20mg pellets). At the start of a trial, the mouse was placed on the central platform, and after $15 \mathrm{sec}$, the doors giving access to the first pair of selected arms were opened simultaneously. Once the mouse had reached the end of the chosen arm (baited or not) it was allowed to return to the central platform, where it was again confined. After $15 \mathrm{sec}$, the same two doors were opened for the next trial. Training continued until the animal reached a criterion of 13 correct choices in the same 16-trial session or 12 correct choices out of 16 on two successive sessions. Training for the second problem, A2, began the next day and continued until animals attained the same criterion of performance. The following day an additional session was given in which both pairs $(\mathrm{A} 1+\mathrm{A} 2)$ were presented in a mixed order. This last session, aimed at familiarizing mice with the mixed-testing procedure to be used postoperatively in Maze B, was given 6.5 weeks before the beginning of hippocampal seizure induction. Two weeks later ( 4.5 weeks before treatment), animals were trained on the first pair (B1) in Maze B; they were subsequently trained on Pairs B2 and B3, respectively, about 2.5 weeks and 4 days before the onset of hippocampal seizures. The position of the baited arms was counterbalanced across successive pairs, mazes, and subjects, thus precluding the use of simple side strategies to solve the spatial discrimination problems.

Posttreatment testing. The degree of retention and the rate of reacquisition of the pairs learned before the beginning of treatment in Maze B (B1, B2, B3) were assessed as follows. During each daily session, the three different pairs were presented, eight times each, in a mixed order. Upon completion of testing in Maze B, animals were given a single-retention session for the two pairs (A1, A2) learned in Maze A, each being presented eight times in a mixed order. The percentages of correct responses recorded both on the first 8 trials (first session) and on the first 16 trials (first two sessions) were used as retention scores. Moreover, the number of sessions needed to reattain the $13 / 16$ criterion was calculated for each problem in Maze B. Retention scores in Maze A were calculated by the percentage of correct responses recorded both on the first 8 trials and over the 16 trials of the single-retention session.

\section{Hippocampal stimulation}

Surgery. Implantation of electrodes was conducted 1 week following isolation. Animals were deeply anesthetized with an intraperitoneal injection of sodium thiopental $(100 \mathrm{mg} / \mathrm{kg})$ and positioned in a Kopf stereotaxic apparatus. After trepanation of the skull, bipolar electrodes were positioned bilaterally so that the tips were located near the CA1 pyramidal cells of the right and left hippocampi. Each bipolar electrode was made up of two platinum wires, $90 \mu \mathrm{m}$ in diameter, which were tightly twisted together and insulated except for the tip. A silver wire was used as reference background. The two electrodes and the silver wire were connected to a miniature connector. Three jeweler's screws were screwed to the skull and the entire electrode apparatus was fixed with dental cement. The stereotaxic coordinates of CAI field in dorsal hippocampus were $1.7 \mathrm{~mm}$ posterior to the bregma, $1.3 \mathrm{~mm}$ lateral from the midline, and $1.75 \mathrm{~mm}$ below the calvarium.

Stimulation parameters. Depending on the intensity of posttraining hippocampal stimulation used, symmetrically opposite effects are observed on retention test performance (Destrade, 1979). As a general rule, posttraining electrical stimulation at or above the $\mathrm{AD}$ induction threshold disrupts subsequent retention performance in animals (Kesner \& Wilburn, 1974; Mellanby, Green, Impey, Oates, \& Traynor, 1984; Olton \& Wolf, 1981) as well as in humans (Halgren \& Wilson, 1985).

Afterdischarge threshold. Ten days after surgery, individual AD threshold values were determined. Each animal was placed in a wooden box situated in a grounded wire mesh cage. Each hippocampal electrode was connected to the stimulator and to the recording apparatus (polygraph). The electrical activity of each hippocampus (theta rhythm) was recorded in order to verify that activity patterns were normal ( $100 \mu \mathrm{V}$ in amplitude and 8-9 cycles/sec in frequency). Each animal was subjected daily to a single stimulation. A 4-sec train of $100-\mathrm{Hz}$ sinusoidal current was presented (stimulation train of $200 \mathrm{msec}$ separated by $200 \mathrm{msec}$ ) simultaneously to the two hippocampi. Stimulation began at $10 \mu \mathrm{A}$. If the subject did not display AD at this level, the intensity was increased in increments of $5 \mu \mathrm{A}$ on each following day until a bilateral $\mathrm{AD}$ was evoked. Behavioral disturbance (arrest reaction) observed during stimulation and during afterdischarge was slight.

Postacquisition treatment. Twenty-four hours following acquisition of the last problem acquisition, 12 implanted animals were stimulated bilaterally at an intensity sufficient to produce electrophysiological seizures. Stimulations were delivered four times, $1 \mathrm{~h}$ apart, and elicited ADs on each stimulation. The other 11 implanted mice were placed in the wooden box without receiving any stimulation.

\section{Histology}

At the end of testing, each animal was decapitated; the brain was removed, fixed in formol $(10 \%)$ and formol saccharose, frozen, and sectioned horizontally on a microtome at $80 \mu \mathrm{m}$. Brain sections were stained using thionin for histological verification of electrode position in both hippocampi.

\section{RESULTS}

\section{Histology}

The tips of each bipolar electrode of the 12 stimulated mice (Figure 2 ) were correctly located near the CAl pyramidal cells of each hippocampus.

\section{Afterdischarge Thresholds}

The mean value of the AD threshold was $21.7 \pm$ $3.0 \mu \mathrm{A}(n=12)$. Stimulation performed at current levels inducing $\mathrm{ADs}$ had a dramatic effect on theta wave recorded immediately following the stimulation; several 


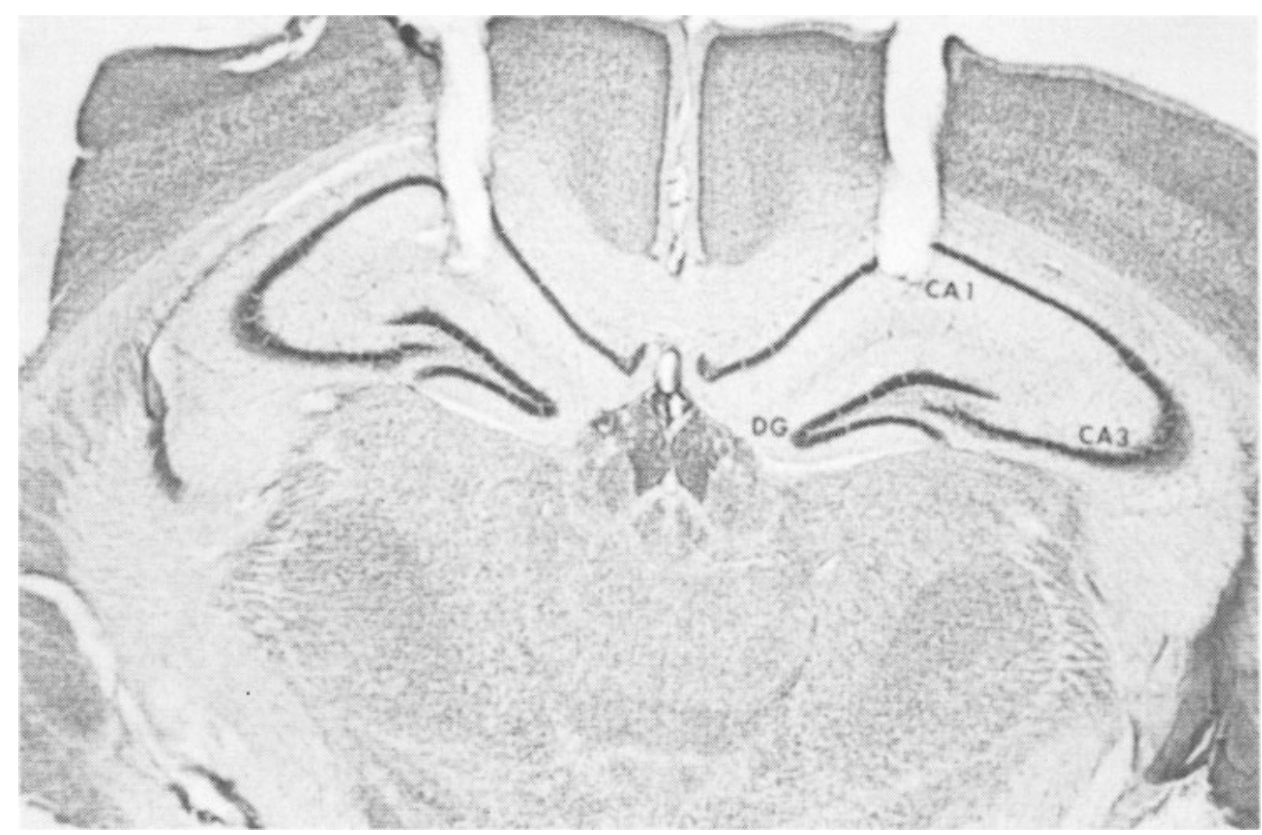

Figure 2. Frontal section showing the electrode placement in CA1 field of dorsal hippocampus.

high-amplitude (1-2 V) spikes occurred during the next $20 \mathrm{sec}$. Subsequently, the amplitude of the records decreased markedly to become virtually a flat line. Four to five minutes after the end of the stimulation, the amplitude of the electroencephalographic (EEG) records began to increase. Electrical disturbance measured during and after hippocampal AD lasted for about $5 \mathrm{~min}$ (Figure 3). No evidence of kindling or a potentiation of the seizure- producing effect of the stimulation was observed during the course of the experiment.

\section{Behavior}

Acquisition of each spatial discrimination problem.

All mice learned the discrimination problems accurately.

They learned the first pair (Maze A) in an average of 5.83 sessions. The subsequent four pairs (A2, B1, B2,

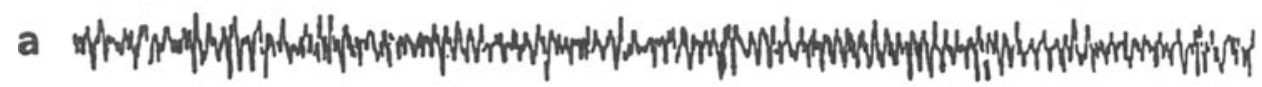

( $200 \mu \mathrm{V}$

\section{b}

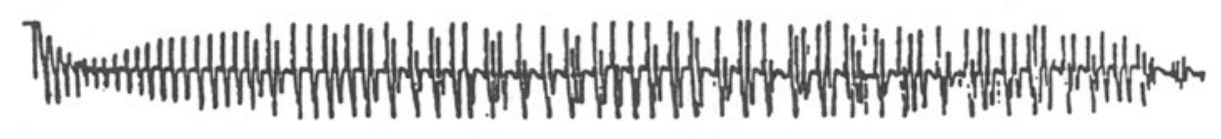

[ $200 \mu \mathrm{V}$

C

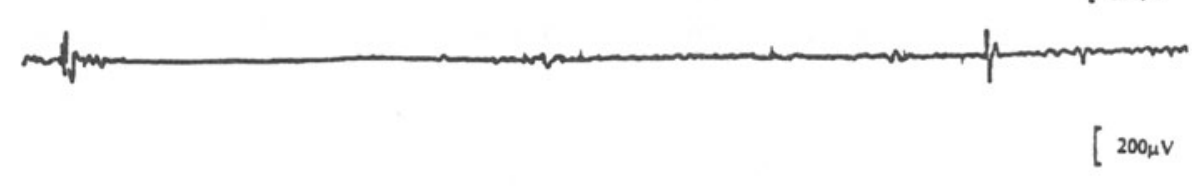

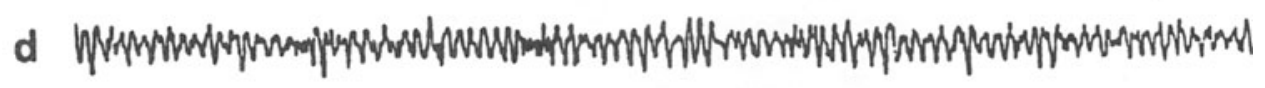

Figure 3. Polygraph recording from an effective stimulation of dorsal hippocampus. (a) Theta wave recording before hippocampal stimulation (bioelectrical activity 4-8 $\mathrm{Hz}$ in frequency and about $200-300 \mu V$ in amplitude). (b) Immediately following the stimulation $(25 \mu \mathrm{A})$, several high-amplitude (1-2 V) spikes occurred during the next 20 sec. (c) Subsequently, the amplitude of the recordings decreased markedly to a flat line. (d) About 5 min after stimulation, slow wave recordings returned to normal. 
Table 1

Acquisition Scores (Mean Percentage Correct) on the Last Daily Session for Each of the Five Discrimination Problems Learned in Both Maze $A$ (A1 and A2) and Maze B (B1, B2, B3) in Stimulated, Implanted, and Nonimplanted Groups

\begin{tabular}{llllllll}
\hline & \multicolumn{3}{c}{ Maze A Pair } & \multicolumn{3}{c}{ Maze B Pair } \\
\cline { 2 - 8 } \multicolumn{1}{c}{ Group } & A1 & A2 & A1 + A2 & B1 & B2 & B3 \\
\hline Stimulated $(n=12)$ & 80 & 83 & 71.5 & 86.6 & 89.3 & 86.6 \\
Implanted $(n=11)$ & 83.5 & 83.5 & 71 & 84.6 & 88 & 88 \\
Nonimplanted $(n=7)$ & 83.8 & 83.8 & 76.5 & 88 & 89.3 & 84.4 \\
\hline
\end{tabular}

B3 ) were learned more rapidly, respectively-3.14 (A2), $2.86(\mathrm{~B} 1), 2.46(\mathrm{~B} 2)$, and 2.73 (B3) sessions. The mice showed an improvement in problem learning [trend analysis on the five pairs, $F(4,29)=38.9, p=.0001]$. Specifically, the number of sessions needed to achieve criterion reduced progressively as the number of problems solved increased, but this number did not differ among the animals for the total of pairs learned $[F \mathrm{~s}<1]$. During the last session of each discrimination learning, mice reached comparable performance levels ranging from $80 \%$ to $89.3 \%$ of correct responses $(F \mathrm{~s}<1$, see Table 1). When the mice were tested concurrently on pairs $\mathrm{A} 1+\mathrm{A} 2$, response accuracy decreased with respect to previous performance achieved for each separate pair, but this did not differ among the three groups $[F(2,27)<$ $0.66 ; p>.50]$.

Retrograde amnesia. Retention performance for the spatial discriminations learned 7 weeks $(\mathrm{Al}+\mathrm{A} 2)$, 4.5 weeks (B1), 2.5 weeks (B2), and 3 days (B3), respectively, before hippocampal stimulation is summarized in Figure 4. Nonimplanted animals $(n=7)$ and implanted but nonstimulated animals $(n=11)$ displayed comparable retention performance levels for the five pairs $(F \mathrm{~s}<1)$, so these two groups have been pooled (control group $n=18$ ) for statistical analysis.

In terms of retention scores in Maze B, control mice exhibited significant forgetting of the discriminations over time. From $85.8 \%$ for the third pair (B3), learned 10 days earlier, discrimination scores decreased to $74.3 \%$ for the first one (B1), learned 5.5 weeks before testing [trend analysis; first 8 trials, $F(2,34)=7.45, p=$ .002 ; first 16 trials, $86.1 \%-71.5 \%, F(2,34)=3.81, p=$ $.03]$. In contrast, the discrimination scores of experimental subjects $(n=12)$ were not significantly different among the three pairs either for the first 8 or for the total of 16 trials $\left(F_{\mathrm{s}}<1\right)$. However, although response accuracy for each pair was not significantly different from chance levels $(50 \%)$ on the first 8 trials $[t s(11)<1.1$, $p>.30]$, it increased significantly above chance level for the next 8 trials $[t \mathrm{~s}(11)>2.42, p<.05]$. Finally, as compared with control mice, all stimulated animals exhibited a dramatic and significant impairment of response accuracy for problems learned 10 days (B3), 3.5 weeks (B2), and 5.5 weeks (B1) earlier, whatever number of trials was considered [ 8 first, $F(1,28)>9.3$, $p<.01 ; 16$ first trials, $F(1,28)>11.7, p<.01]$.

In terms of retention scores in Maze A, scores for the pairs $(\mathrm{A} 1+\mathrm{A} 2)$, learned 7 weeks before hippocampal stimulation, were not significantly different for the experimental and control groups either for the first 8 trials [respectively, $64.6 \pm 8.5 \%$ and $59.7 \pm 5.6 \%$ ] or the 16 trials $[69.3 \pm 5.3 \%$ vs. $64.9 \pm 3.9 \% ; F(1,28)=0.45$, $p>.50$ for both comparisons].

As shown in Figure 5, a comparison of the mean retention scores displayed by the experimental and control groups in Maze A ( $\mathrm{Al}+\mathrm{A} 2)$ and Maze B (B1 + B2 + B3) for the first eight trials showed the following: Whereas control animals exhibited significant forgetting for pairs learned 6-7 weeks before (Maze A) compared with pairs learned 0.5-4.5 weeks before (Maze B; $78 \%-64.9 \%$ ), an inverse pattern of results was observed for experimental subjects [55.9\%-69.3\%; two-way analysis of variance, $F(1,27)=15.5, p<.001]$.

Reacquisition in Maze B. The mean number of sessions needed to reacquire the 13/16 criterion on each of the three pairs (B1, B2, B3) tested concurrently (eight trials per pair on each session) was significantly higher for the experimental group (4-5 sessions) than for both control (implanted and nonimplanted) groups $[F(2,29)>$ $5.58, p<.01$; post hoc tests using Scheffé $F$ tests, $p$ s $<$ .05 ; see Figure 6]. Experimental animals required the same amount of retraining as for initial acquisition regardless of which B pair was considered $[t \mathrm{~s}(11)<1.603$, $p>.137]$. Furthermore, the attainment of the reacquisition criterion was not significantly different among the three pairs [ts $(11)<1.48, p>.16$ ], suggesting again a comparable degree of memory loss for each of these three problems.

\section{DISCUSSION}

The present experiment shows that the repeated posttraining induction (four times, $1 \mathrm{~h}$ apart) of hippocampal ADs significantly impaired the retention of two-choice

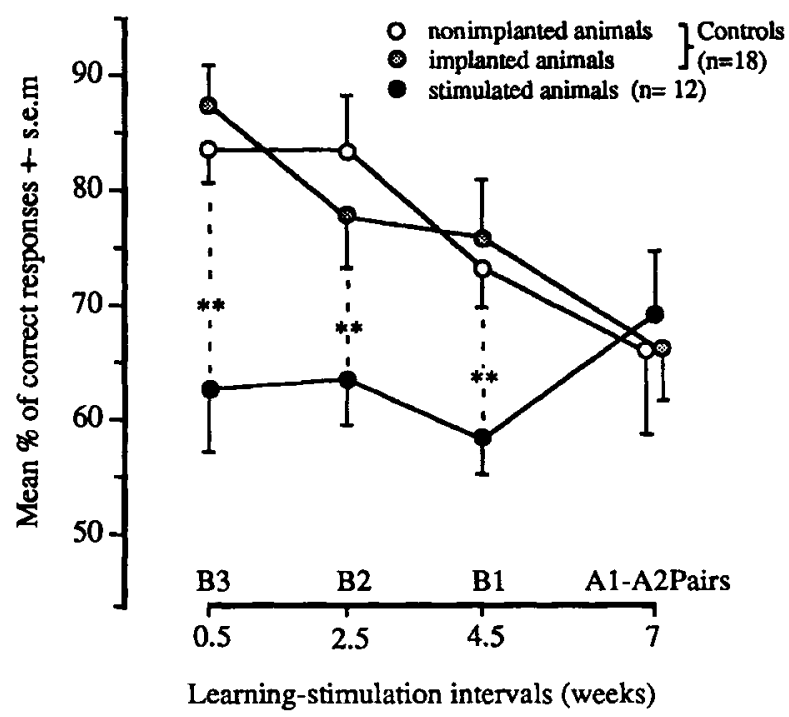

Figure 4. Retention scores on the first 16 trials $(M \pm S E M)$ in stimulated, implanted, and nonimplanted animals, as a function of learning-hippocampal stimulation interval. **p $<.01$. 


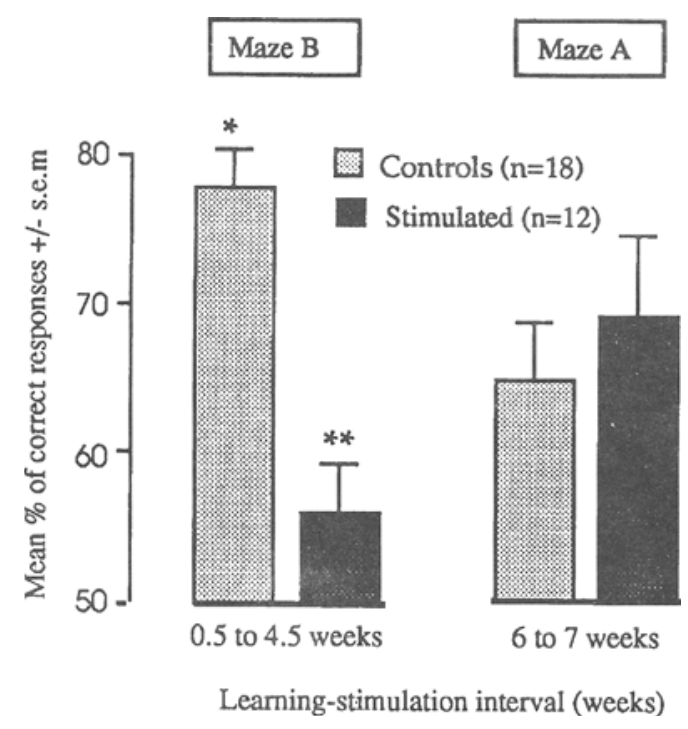

Figure 5. A comparison between retention scores on the first eight trials in Maze B (B1 + B2 + B3) and Maze A $(A 1+A 2)$. For control animals, retention scores were better in Maze $B\left({ }^{*} p<\right.$ $.05)$, whereas stimulated animals exhibited an inverse pattern of results. $\left({ }^{* *} p<.01\right)$.

discrimination problems learned between about 4 days to 4.5 weeks prior to treatment, but did not alter the retention of problems learned 7 weeks earlier. As we hypothesized in the introduction, these data indicate that the susceptibility of the memory trace to disruption by repeated hippocampal seizures is indeed temporally graded, leaving intact the more remotely acquired ones. It must be noted, however, that the present findings provide no firm evidence for the existence of such a gradient among the three different discrimination problems, each learned at different time periods in the same maze (B). Specifically, although control mice exhibited significant forgetting with increasing retention intervals (from about 10 days to 6 weeks), hippocampal AD-treated mice exhibited poor retention performance no matter how much time had elapsed between learning and treatment (i.e., 0.5, 2.5, and 4.5 weeks; see Figure 4). Thus, using the same behavioral protocol as that used by Cho et al. (1993), we did not observe, across the same 4.5-week time period, the temporally graded RA previously reported by these authors following entorhinal cortex lesions.

Taken together, the present findings raise several questions that will be discussed in turn. The first question is whether the observed impairment of retention scores in Maze B in experimental subjects truly reflects an extended RA and, if so, whether this RA may be specifically attributed to the transient hippocampal dysfunction induced by electrical stimulation. One aim of the present experiment was to use a reversible disruption of hippocampal functioning in order to distinguish between the roles of HF in consolidation and subsequent retrieval of the acquired information. Thus, the underlying assumption was that hippocampal function would be normal during retention testing. One might suppose, however, that such electrical stimulation would induce a more or less long-lasting hippocampal dysfunction, and that this proactive effect is responsible for the retrieval deficit. Two arguments weigh against this hypothesis. First, although experimental animals displayed chancelevel performance at the beginning of retention testing in Maze B (first eight trials), they were nevertheless able to subsequently improve their performance at about the same rate as during initial acquisition. Second, this hypothesis seems unable to account for the total lack of effect of the treatment on retention scores in Maze A. One might also suppose that the presently observed impairment of retention scores in Maze B was not necessarily specific to the transient hippocampal dysfunction induced by electrical stimulation, since hippocampal ADs might have spread out of the hippocampal formation, especially through neocortical areas, which are thought to be the repository of permanent memory traces. If this were indeed the case, experimental subjects should also have exhibited impaired retention scores for pairs learned in Maze A.

The second question that deserves consideration concerns the lack of a temporal gradient of RA for pairs learned in Maze B. Specifically, our results show that, for the two pairs learned in Maze A, experimental animals not only exhibited the same retention scores as controls, but also displayed even better retention scores than for the three more recently learned pairs in Maze B. Although this result would indicate that, beyond an approximate 7-week period, memory for pairs became completely resistant to transient hippocampal dysfunction, it is not clear why there was no evidence at all for a temporal gradient of RA for pairs learned across the preceding 4.5-week period (Maze B). The consolidation process is generally conceived of as the gradual development, via a hippocampo-neocortical dialogue, of neocortical

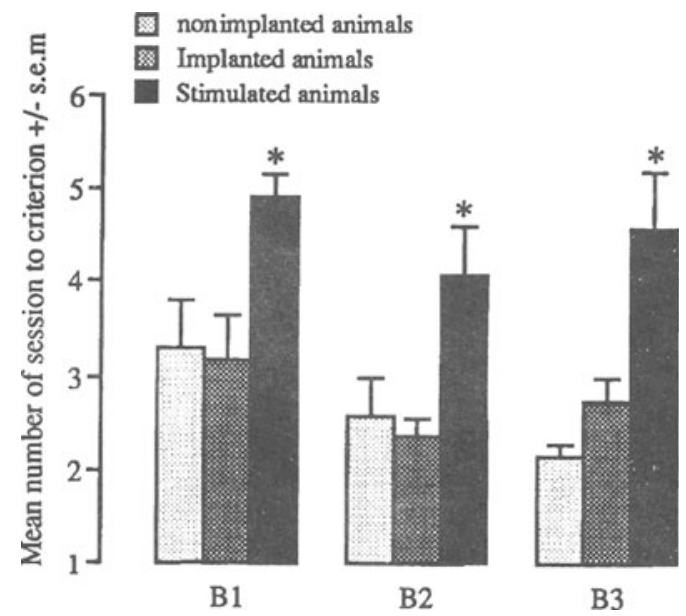

Figure 6. Number of sessions (eight trials per session) needed to reachieve the $13 / 16$ criteria on pairs $B 1, B 2$, and $B 3$, learned respectively $4.5,2.5$, and 0.5 weeks before treatment in the nonimplanted, implanted, and stimulated groups. ${ }^{*} p<.05$. 
engrams that can eventually sustain by themselves the recall of information, the initial acquisition of which depends predominantly on the hippocampal system (see Squire \& Alvarez, 1995). In particular, Miller (1989) envisioned the existence of a dynamic interplay between the neocortex and hippocampus via the existence of abundant reciprocal pathways and assigned to the hippocampal theta rhythm the role of an oscillator permitting the establishment of a resonant loop with the neocortex, which is necessary for long-term engrammation into cortical cell assemblies of plurimodal (contextual) information in general. Most importantly, Miller assumed that the resonating loop is necessary for coordinating representations of the environment, or contexts, in which specific events or target information may be distinguished and, thus, specifically retrieved. A possible explanation for the lack of a temporal gradient of RA for pairs learned in Maze B emerges directly from these considerations. Indeed, our data suggest that the deleterious retroactive effects of hippocampal stimulation on the retention of spatial information are the same whatever the delay, insofar as the information is acquired in the same context (Maze B). It is widely accepted that animals learn about the spatial arrangement of target stimuli (reinforced arms) by forming cognitive maps (O'Keefe \& Nadel, 1978) or representations involving configural relationships among cues that are present at the time of learning (Eichenbaum, Otto, \& Cohen, 1992; Sutherland $\&$ Rudy, 1989). Thus, hippocampal ADs may have erased or disorganized the hippocampal representations shared by, and underlying, each of the learned discriminations. More precisely, in the present experimental situation, there were obvious extramaze cues that remained constant over learning sessions and that constituted a common feature of each learning episode in Maze B. Thus, it can be hypothesized that learning the third problem in Maze B (B3) would have, at least partially, reactivated the memory of the previously learned pairs (B1, B2) and reinstated the susceptibility of these more or less consolidated memory traces to disruption. Indeed, certain experiments have provided evidence that, although remote information is relatively resistant to amnestic insults, amnesia can nevertheless be induced if the earlier learning episode is cued or reactivated prior to the delivery of electroconvulsive shock (Misanin, Miller, \& Lewis, 1968) or other amnestic treatments (Bucherelli \& Tassoni, 1992; Mactutus, Riccio, \& Ferek, 1979; Richardson, Riccio, \& Mowrey, 1982).

It is thus possible that in the present experiment, as well as in the experiment of Gaffan (1993), in which monkeys were subjected to a single-retention session of remotely acquired material (6 months) 3 days before surgery (fornix lesions), the lack of temporal gradient for the observed extensive RA may be explained by a reactivation of these more remotely acquired memories. More simply, the problem may be the following: To the extent that each learning episode (B1-B3) occurred within the same context and therefore remained dependent on the same spatial index, our amnestic treatment may have affected predominantly memories sharing the same contextual characteristics or features. Indeed, it seems evident that, in our experimental design, the most recently acquired spatial information (B3) may not have been encoded as an isolated entity but rather in relation to previous information ( $\mathrm{B} 1$ and $\mathrm{B} 2$ ), as part of a cognitive map. In line with this idea, Robbins and Meyer (1970) showed that two-choice discriminations learned under the same motivational "context" as those prevailing at the time of treatment are impaired, and that these effects are independent of the age of the problem being tested for retention. Together, these explanatory alternatives are not mutually exclusive and outline the dynamic aspects of postacquisition information processing, as revealed by our treatment.

Furthermore, it must be noted that the acquisition and retention procedure used for $\mathrm{B}$ pairs raises the problem of interference. Indeed, the fact that the three spatial discriminations in Maze B were acquired successively and were required to be remembered concurrently by the same experimental subjects is not without problems. This shortcoming could be avoided by the use of independent groups for each retention interval. Finally, it is questionable whether the total lack of impairment of retention for pairs acquired in Maze A may be entirely accounted for by the time interval between acquisition and treatment. Indeed, in addition to being learned 7 weeks before hippocampal AD treatment, these two pairs were also the ones for which animals received the highest degree of training as compared with that required for the following B pairs (for which, nevertheless, subjects displayed higher levels of choice accuracy by the end of training; see Table 1). Thus, one cannot rule out the possibility that the sparing of memory for discriminations learned in Maze A results from the length of the initial acquisition period, rather than from the age of memory per se (see also Bolhuis et al., 1994). In other words, the question arises whether such an extensive period of training does not render the memory trace less dependent on the HF for subsequent consolidation-that is, whether a part of the consolidation process does not actually occur during the training session itself. On this reasoning, repetition of the same event would provide the opportunity for a rapid incremental adjustment of neocortical connections, thereby allowing memories initially dependent on the hippocampal system to gradually become independent of it (corticalization of memory traces). Obviously the present data do not permit an unequivocal reply regarding the relationships between the amount of training, different degrees of forgetting, and the occurrence of a time-dependent gradient of RA.

The hypothesis of a temporally limited involvement of the HF in retrieval processes seems tenable, since we observed preserved retention scores at longer delays ( 7 weeks) and impairments at more recent ones (4.5-0.5 weeks). However, the possibility that this gradient resulted from either a change in the context of learning (Maze B vs. Maze A) rather than to time itself cannot be excluded at present. The lack of context specificity of each spatial dis- 
crimination (Maze B) may have overshadowed a timedependent effect of the HF treatment. In conclusion, in order to clarify and extend our results, future studies should vary the amount of information to be remembered in a given physical context. The best alternative would be to individualize each spatial discrimination problem by assigning to it a distinct temporal and physical context.

\section{REFERENCES}

Bolmuis, J. J., Stewart C. A., \& Forrest, E. M. (1994). Retrograde amnesia and memory reactivation in rats with ibotenate lesions to the hippocampus or subiculum. Quarterly Journal of Experimental Psychology, 47, 129-150.

Bucherelli, C., \& Tassoni, G. (1992). Engram activation reinstates the susceptibility of consolidated memory traces to retrograde amnesia by functional blockade of parabrachial nuclei. Behavioural Brain Research, 51, 61-65.

Cho, Y. H., Béracochéa, D., \& Jaf̧Fard, R. (1993). Extended temporal gradient for the retrograde and anterograde amnesia produced by ibotenate entorhinal cortex lesions in mice. Journal of Neuroscience, 13, 1759-1766.

CHO, Y. H., \& KESNER, R. P. (1996). Involvement of entorhinal cortex in long term spatial discrimination memory in rats: Retrograde amnesia. Behavioral Neuroscience, 110, 436-442.

DESTRADE, C. (1979). Le phénomène de réminiscence chez la souris: Conditions normales d'apparition et effets de certaines stimulations électriques centrales. Unpublished thesis, Université de Bordeaux I.

Eichenbaum, H., Otto, T., \& Cohen, N. J. (1992). The hippocampus, what does it do? Behavioral \& Neural Biology, 57, 2-36.

GAFFAN, D. (1993). Additive effects of forgetting and fornix transection in the temporal gradient of retrograde amnesia. Neuropsychologia, 31, 1055-1066.

Halgren, E., \& Wilson, C. L. (1985). Recall deficits produced by afterdischarges in the human hippocampal formation and amygdala. Electroencephalography \& Clinical Neurophysiology, 61, 375-380.

KESNER, R. P., \& WILBURN, M. W. (1974). A review of electrical stimulation of the brain in context of learning and retention. Behavioral Biology, 10, 259-293.

KiM, J. J., CLARK, R. E., \& Thompson, R. F. (1995). Hippocampectomy impairs the memory of recently, but not remotely, acquired trace eyeblink conditioned responses. Behavioral Neuroscience, 109, 195 203.

Kim, J. J., \& FANSELOW, M. S. (1992). Modality-specific retrograde amnesia of fear. Science, 256, 675-677.

MACTUtUs, C. F., Riccio, D. C., \& Ferek, J. M. (1979). Retrograde amnesia for old (reactivated) memory: Some anomalous characteristics. Science, 204, 1319-1320.
Mellanby, J., Green, A. R., Impey, L., Oates, C., \& Traynor, L. (1984). The effect of electroconvulsive shock on learning and memory in rats. Acta Neurologica Scandanavica, 69, 115-118.

MiLLER, R. (1989). Cortico-hippocampal interplay: Self-organizing phase-locked loops for indexing memory. Psychobiology, 17, 115128.

Misanin, J. R., Miller, R. R., \& Lewis, D. J. (1968). Retrograde amnesia produced by electroshock after reactivation of a consolidated memory trace. Science, 160, 554-555.

NADEL, L. (1991). The hippocampus and space revisited. Hippocampus, 1, 221-229.

O'KeEFE, J., \& NADEL, L. (1978). The hippocampus as a cognitive map. Oxford: Oxford University Press, Clarendon Press.

Olton, D. S., \& Wolf, W. A. (1981). Hippocampal seizures produce retrograde amnesia without a temporal gradient when they reset working memory. Behavioral \& Neural Biology, 33, 437-453.

RichaRdSON, R., Riccio, D. C., \& Mowrey, H. (1982). Retrograde amnesia for previously acquired Pavlovian conditioning: UCS exposure as a reactivation treatment. Physiological Psychology, 10, 384-390.

RoBBins, M. J., \& MEYER, D. R. (1970). Motivational control of retrograde amnesia. Journal of Experimental Psychology, 84, 220-225.

Salmon, D. P., Zola-Morgan, S., \& Squire, L. R. (1987). Retrograde amnesia following combined hippocampal-amygdala lesions in monkeys. Psychobiology, 15, 37-47.

SQuire, L. R. (1992). Memory and the hippocampus: A synthesis from findings with rats, monkeys, and humans. Psychological Review, 99, 195-231.

SQUiRE, L. R. (1994), Memory and forgetting: Long term and gradual changes in memory storage. International Review of Neuroscience, 17, 243-269.

SQuiRE, L. R., \& ALVAREZ, P. (1995). Retrograde amnesia and memory consolidation: A neurobiological perspective. Current Opinion in Neurobiology, 5, 169-177.

SquiRE, L. R., \& SPANIS, C. W. (1984). Long gradient of retrograde amnesia in mice: Continuity with the findings in humans. Behavioral Neuroscience, 98, 345-348.

SutherLand, R. J., \& Rudy, J. W. (1989). Configural association theory: The role of the hippocampal formation in learning, memory, and amnesia. Psychobiology, 17, 129-144.

Teyler, T. J., \& DiScenNa, P. (1986). The hippocampal memory indexing theory. Behavioral Neuroscience, 100, 147-154.

WINOCUR, G. (1990). Anterograde and retrograde amnesia in rats with dorsal hippocampal or dorsomedial thalamic lesions. Behavioural Brain Research, 38, 145-154.

Zola-Morgan, S. M., \& SQuire, L. R. (1990). The primate hippocampal formation: Evidence for a time limited role in memory storage. Science, 250, 288-290.

(Manuscript received August 6, 1996; revision accepted for publication December 4, 1996.) 